\title{
Magnons in the Heisenberg model with a dynamical scaling symmetry
}

\author{
W.J. Caspers ${ }^{\mathrm{a}, *}$, M. Kuźma ${ }^{\mathrm{a}, 1}$, B. Lulek ${ }^{\mathrm{b}}$, T. Lulek ${ }^{\mathrm{b}}$ \\ ${ }^{a}$ Center for Theoretical Physics, University of Twente, P.O. Box 217, 7500 AE Enschede, Netherlands \\ ${ }^{\mathrm{b}}$ Institute of Physics, Adam Mickiewicz University, Poznań, Poland
}

Received 5 September 1997

\begin{abstract}
A special Heisenberg model is considered for which the exchange integral takes on the same value $J$ not only for geometrically equivalent neighbours, but also for such $j$-neighbours which constitute an orbit of a "hidden" symmetry group of scaling transformations. The dispersion law for magnons for this model constitutes a reproduction of some rules of arithmetic number theory. The extra symmetry is illustrated by a chain of 12 spins, which may be shown to be equivalent to a toroidal $4 \times 3$-periodic crystal. (c) 1998 Elsevier Science B.V. All rights reserved.
\end{abstract}

PACS: 02.20.+b; 03.65.Fd; 75.10.Jm; 75.40.Gb

Keywords: Heisenberg model; Magnons; Dynamic scaling symmetry

\section{Introduction}

It has been established that the model of a finite linear Heisenberg magnet, consisting of $N$ spins $s$, exhibits a scaling symmetry of its kinematical properties [1-3]. Such a symmetry, derived within the recipe of Weyl [4], manifests itself as a fact that the distribution $\rho: B \rightarrow \boldsymbol{Z}$ of quantum states of the magnet over the (finite) Brillouin zone $B$ remains constant on each orbit of action of the hidden symmetry group Aut $C_{N}$ of the linear chain, with the "obvious" symmetry given by the translation group of the crystal, i.e. the cyclic group $C_{N}$. In the present paper we examine some simplest consequences of imposing the scaling transformations as dynamical symmetries of the Heisenberg model. Our principal assumption is that the exchange integral - the main dynamical parameter of the Heisenberg model - takes on the same value $J$ not only for

\footnotetext{
* Corresponding author. Tel.: 053 4893153/4893147; fax.: 053 4891101; e-mail: w.j.caspers@thn.tn. utwente.nl.

${ }^{1}$ On leave from Institute of Physics, Higher Pedagogical School, Rzeszów, Poland.
} 
geometrically equivalent neighbours, but also for all such $j$-neighbours which constitute an orbit of the group Aut $C_{N}$ of scaling transformations. We are going to demonstrate that within such a model the dispersion law for magnons becomes a tool for a nice reproduction of some results of arithmetic number theory, in particular it provides a natural interpretation of quantities like Euler or Möbius function [5] in the realm of finite size effects of the condensed matter theory.

The model considered here mediates between the usual Heisenberg magnet with interactions of nearest neighbours only, and the spherical model in which all neighbours interact with the same exchange integral (see, e.g. Ref. [6]). As we show here, this mediation becomes transparent within a multidimensional crystallographic interpretation of the translational symmetry group $C_{N}$ of a linear ring [7,8]. Moreover, experimental achievements in construction of simple quantum interferometer devices (SQID), and quantum dots, including magnetic ones [9] allow us to express a hope that our model might not be so far from a technological realisation.

\section{The Heisenberg magnetic ring with a dynamical scaling symmetry}

Let $\tilde{N}=\{j=1,2, \ldots, N\}$ be the set of nodes of the magnetic linear ring. We assume it to be a regular orbit of action of the cyclic group $C_{N}-$ the translation symmetry group of the magnet. Let $s$ be the spin of each node, and $\tilde{s}=\{m=s, s-1, \ldots,-s\}$ - the set of all eigenvalues of the $z$-component of the spin operator s. Then the space of all quantum states of the magnet is

$$
L^{\otimes N}=l c_{C} \tilde{S}^{\tilde{N}}
$$

where

$$
\tilde{s}^{\tilde{N}}=\{f: \tilde{N} \rightarrow \tilde{s}\}
$$

is the set of all magnetic configurations, and $l c_{C}$ stands for the linear closure (of the set $\tilde{s}^{\tilde{N}}$ ) over the field $C$ of complex numbers. Thus, the set $\tilde{s}^{\tilde{N}}$ serves as an orthonormal basis in the $N$ th tensor power $L^{\otimes N}$ of the single-node space $L=l c_{C} \tilde{s}$. A magnetic configuration is a mapping $f \in \tilde{s}^{\tilde{N}}$, and can be presented more explicitly as the ket $\left|m_{1}, \ldots, m_{N}\right\rangle$, where $m_{j} \in \tilde{s}, j \in \tilde{N}$.

The action $P: C_{N} \times L^{\otimes N} \rightarrow L^{\otimes N}$ of the cyclic group $C_{N}$ in the space $L^{\otimes N}$ of all quantum states of the magnet is imposed by the formula

$$
P(j)\left|m_{1}, \ldots, m_{N}\right\rangle=\left|m_{(1-j) \bmod N} \ldots, m_{(N-j) \bmod N}\right\rangle, j \in C_{N},\left|m_{1}, \ldots, m_{N}\right\rangle \in \tilde{s}^{\tilde{N}} .
$$

It can be decomposed into irreducible representations (irreps) $\Gamma_{k}$ of the group $C_{N}\left(\Gamma_{k}(j)=\exp (2 \pi i k j / N)\right)$ according to the formula

$$
P \cong \sum_{k \in B} \oplus \rho(k) \Gamma_{k},
$$


where

$$
B=\left\{k=0, \pm 1, \pm 2, \ldots,\left\{\begin{array}{ll} 
\pm(N / 2-1), N / 2 & \text { for } N \text { even } \\
\pm(N-1) / 2 & \text { for } N \text { odd }
\end{array}\right\}\right.
$$

is the (finite) Brillouin zone of the one-dimensional periodic crystal $\tilde{N}$. Decomposition (4) defines the mapping $\rho: B \rightarrow \boldsymbol{Z}$ of the Brillouin zone into the ring $\boldsymbol{Z}$ of integers, given by the multiplicities $\rho(k)$ of $\Gamma_{k}$ in $P$, and referred to as the distribution of quantum states of the magnet over the Brillouin zone $[10,11]$.

The symmetry of the distribution $\rho$ has been thoroughly discussed $[1-3,10,11]$ in terms of the general recipe of Weyl [4]. According to this recipe, the "obvious" translational symmetry of the model implies that some essential features of this model are subjected to the "hidden" symmetry, given by the group

$$
\text { Aut } C_{N}=\left\{\tau_{r}=\left(\begin{array}{c}
j \\
r j \bmod N
\end{array}\right) \mid r \in \tilde{N}, \operatorname{gcd}(r, N)=1\right\},
$$

with gcd denoting the greatest common divisor. Operations $\tau_{r}$ of this group, i.e. automorphisms of the translation group $C_{N}$, are referred to as scaling transformations of the crystal $\tilde{N}$ (by the factor $r$ ). Indeed, this group accounts for a kinematical symmetry of the Heisenberg model, i.e. the symmetry of the distribution $\rho$ of quantum states over quasimomenta. It has been shown that the mapping $\rho$, which is in general inhomogeneous, is exactly constant on each generalized star

$$
B^{\kappa}=\{k \in B \mid \operatorname{gcd}(k, N)=\kappa\} \subset B, \quad \kappa \in K(N),
$$

i.e. on each orbit of the hidden symmetry group Aut $C_{N}$ on the Brillouin zone $B$. Generalized strata are classified by elements $\kappa$ of the set

$$
K(N)=\{\kappa \in \tilde{N} \mid \operatorname{gcd}(\kappa, N)=\kappa\} \subset \tilde{N}
$$

of all divisors of $N$. This set, together with the relation $\leqslant$, defined by

$$
\kappa_{1} \leqslant \kappa_{2} \Leftrightarrow \operatorname{gcd}\left(\kappa_{1}, \kappa_{2}\right)=\kappa_{1}, \quad \kappa_{1}, \kappa_{2} \in K(N)
$$

forms a lattice, i.e. a partially ordered set with unique maximal and minimal element $\kappa=N$ and 1 , respectively.

The Heisenberg - Dirac translationally invariant exchange Hamiltonian of the magnet is

$$
\hat{H}=\sum_{j \in \tilde{N}} \sum_{\delta \in \tilde{N}} J_{\delta} \mathbf{s}_{j} \cdot \mathbf{s}_{(j+\delta) \bmod N},
$$

where $\mathbf{s}_{j}$ is the spin operator of the $j$ th node, and $J_{\delta}$ is the exchange parameter between $\delta$-neighbours, $\delta \in \tilde{N}$. In the following, we put $J_{\delta}=J_{-\delta \bmod N}, J_{N}=0$. The translational symmetry of the model implies that $J_{\delta}$ does not depend upon $j$. There is a variety 
of possible models, compatible with the translational symmetry $C_{N}$. In particular, by putting

$$
J_{\delta}^{\text {geom }} \begin{cases}J & \text { for } \delta= \pm l \leqslant N / 2 \\ 0 & \text { otherwise }\end{cases}
$$

we obtain the model in which only $l$ th neighbours are interacting. The case $l=1$ (nearest-neighbour interactions) is of most frequent use. Clearly, in the model given by Eq. (11), the set $\left\{\delta \in \tilde{N} \mid J_{\delta} \neq\right\}$ constitutes an orbit of the group

$$
C_{1 i}=\left\{\tau_{1}, \tau_{N-1}\right\} \triangleleft \text { Aut } C_{N},(N \geqslant 2),
$$

corresponding to one-dimensional "point" group of geometric symmetry of the ring. Another extreme case is provided by the spherical model, where

$$
J_{\delta}^{\text {sph }}=J, \quad \delta \in \tilde{N} \backslash\{N\} .
$$

This model is invariant under the whole group Aut $C_{N}$ of scalings.

Clearly, an arbitrary distribution of values of the exchange parameter $J_{\delta}, \delta \in \tilde{N}$, preserves the obvious symmetry of the recipe of Weyl, but, in general, breaks the hidden symmetry Aut $C_{N}$. Now, we propose the most general form of the Heisenberg Hamiltonian, which is invariant under both groups, $C_{N}$ and Aut $C_{N}$. For such a Hamiltonian, scaling transformations $\tau_{r} \in$ Aut $C_{N}$ are symmetries of not only kinematics, but also of dynamics of the Heisenberg magnet.

Let

$$
\tilde{N}=\bigcup_{\kappa \in K(N)} \tilde{N}^{(\kappa)}
$$

be a (disjoint) decomposition of the set $\tilde{N}$ of nodes of the magnet into subsets

$$
\tilde{N}^{(\kappa)}=\left\{\xi \frac{N}{\kappa} \mid \operatorname{gcd}(\xi, \kappa)=1\right\} \subset \tilde{N}, \quad \kappa \in K(N),
$$

so that

$$
\tilde{\kappa}^{(1)}=\{\xi \in \tilde{\kappa} \mid \operatorname{gcd}(\xi, \kappa)=1\} \subset \tilde{\kappa}
$$

is the set of all positive integers smaller than $\kappa$ and mutually prime with $\kappa$. Clearly, the sets $\tilde{N}^{(\kappa)}, \kappa \in K(N)$, are orbits of the group Aut $C_{N}$ acting on the crystal $\tilde{N}$. Then

$$
\hat{H}=\sum_{\kappa \in K(N)} J_{\kappa}^{\mathrm{sc}} \sum_{\delta \in \tilde{N}^{(k)}} \sum_{j \in \tilde{N}} \mathbf{s}_{j} \cdot \mathbf{s}_{(j+\delta) \bmod N}
$$

is the most general form of the scaling-invariant Heisenberg Hamiltonian for the ring $\tilde{N}$. Any one-parameter version of the scaling-invariant Hamiltonian is specified by

$$
J_{\delta}^{(\kappa)}= \begin{cases}J & \text { for } \delta \in \tilde{N}^{(\kappa)}, \\ 0 & \text { otherwise }\end{cases}
$$

i.e. by the choice of a single subset $\tilde{N}^{(\kappa)}$ in the decomposition (14). 


\section{The dispersion law for magnons}

The ground state of the Hamiltonian (17) with the exchange parameters (18) is ferromagnetic for $J<0$. Magnons are those excitations from the ferromagnetic saturation configuration

$$
|\operatorname{vac}\rangle=|s, \ldots, s\rangle
$$

which correspond to all states with a single spin deviation.

Let

$$
|j\rangle=|s, \ldots, s-1, \ldots, s\rangle, \quad j \in \tilde{N}
$$

be the configuration in which the deviation is localised at the node $j \in \tilde{N}$. All states of the form (20) constitute a regular orbit of the group $C_{N}$ acting on the set $\tilde{n}^{\tilde{N}}$, and thus span an invariant subspace $L_{(1)}^{\otimes N}$ of the space $L^{\otimes N}$. Clearly, this space is also invariant with respect to the Hamiltonian (17), and each eigenstate

$$
|k\rangle=N^{-1 / 2} \sum_{j \in \tilde{N}} e^{2 \pi i k j / N}|j\rangle, \quad k \in B,
$$

describes the magnon with the quasimomentum $k$. One can readily obtain that

$$
\hat{H}|k\rangle=E(k)|k\rangle, \quad k \in B,
$$

with the eigenvalue

$$
E(k)=\sum_{\delta \in \tilde{N} \backslash\{N\}} J_{\delta} s\left(N s-2+e^{-2 \pi i k \delta / N}+e^{2 \pi i k \delta / N}\right) .
$$

We use the formula (cf., e.g. Ref. [10])

$$
w_{N}(k, \kappa)=: \sum_{\delta \in \tilde{N}^{(k)}} e^{2 \pi i k \delta / N}=\frac{\varphi(\kappa)}{\varphi\left(\kappa^{\prime}\right)} \mu\left(\kappa^{\prime}\right),
$$

where $\varphi$ and $\mu$ is the Euler and Möbius function of the number theory, specified by the formulas

$$
\varphi(N)= \begin{cases}1 & \text { for } N=1 \\ N & \prod_{p \in \pi(N)}(p-1) / p\end{cases}
$$

and

$$
\mu(N)= \begin{cases}1 & \text { for } N=1, \\ (-1)^{|\pi(N)|} & \text { for } \alpha_{p}=1, \quad p \in \pi(N) \\ 0 & \text { otherwise, }\end{cases}
$$


with

$$
N=\prod_{p \in \pi(N)} p^{\alpha_{p}(N)}
$$

being the canonical decomposition of $N$ into primes $p, \pi(N) \subset K(N)$ denoting the socle of $N$, i.e. the set of all primes $p \in K(N)$, and

$$
\kappa^{\prime}=\frac{\kappa}{\operatorname{gcd}(k, \kappa)} .
$$

Eq. (24) is a trigonometric result emerging from the group endomorphism $\psi_{k}: C_{N} \rightarrow$ $C_{N}$, given by

$$
\psi_{k}(\delta)=k \delta \bmod N, \quad \delta \in \tilde{N} .
$$

The set $\tilde{N}^{(\kappa)}$, i.e. the orbit of Aut $C_{N}$ on $C_{N}$, is mapped under this endomorphism onto the set $\tilde{N}^{\left(\kappa^{\prime}\right)}=\operatorname{Im}_{\psi_{k}} \tilde{N}^{(\kappa)}$, with the reduced divisor $\kappa^{\prime}$ given by Eq. (28). The Euler functions $\varphi(\kappa)$ and $\varphi\left(\kappa^{\prime}\right)$ give the number of elements in the set $\tilde{N}^{(\kappa)}$ and $\tilde{N}^{\left(\kappa^{\prime}\right)}$, respectively, whereas the Möbius function $\mu\left(\kappa^{\prime}\right)$ accounts for the summation over appropriate phase factors in Eq. (24).

Eq. (24) yields the following general form for energies of magnon states for an arbitrary scaling-invariant Heisenberg Hamiltonian:

$$
E(k)=\sum_{\kappa \in K(N)} J^{(\kappa)} s \varphi(\kappa)\left[N s-2+2 \frac{\mu\left(\kappa^{\prime}\right)}{\varphi\left(\kappa^{\prime}\right)}\right], \quad k \in B .
$$

The dispersion law for scaling-invariant magnons is thus expressed in terms of quotients of Euler functions, modulated by Möbius functions, defined on the lattice $K(N)$ of divisors of $N$.

For a one-parameter version (18) of the scaling-invariant Heisenberg Hamiltonian, energies of magnon states vary around the value $J_{s}(N s-2) \varphi(\kappa)$, which corresponds to vanishing of the Möbius function $\mu\left(\kappa^{\prime}\right)$. The sign of this variation is determined by the reduced divisor $\kappa^{\prime}$, and its amplitude - by the quotient $1 / \varphi\left(\kappa^{\prime}\right)$.

\section{Multidimensional realization of the linear ring}

A regular orbit of the cyclic group $C_{N}$ can be realised geometrically as a multidimensional crystal, along the canonical decomposition (27) of $N$ into prime factors $[7,8]$. Let

$$
N_{p}=p^{\alpha_{p}(N)}, \quad p \in \pi(N)
$$

be the Sylow factor of $N$, corresponding to the prime divisor $p \in \pi(N) \subset K(N)$, so that $C_{N}$ can be factorised into Sylow subgroups

$$
C_{N} \cong \prod_{p \in \pi(N)} \times C_{N_{p}}=: A
$$


Accordingly, we have

$$
\tilde{N} \cong \prod_{p \in \pi(N)} \times \tilde{N}_{p}
$$

so that the set $\tilde{N}$ of nodes of the linear chain is presented as a $|\pi(N)|$-dimensional periodic crystal, with cartesian axes labelled by primes $p \in \pi(N)$, and Born-Kármán periods $N_{p}$ along the axis $p$. The multidimensional counterpart of the label $j \in \tilde{N}$ of the linear ring can be written in a "vector" form

$$
\mathbf{j}=\sum_{p \in \pi(N)} j_{p} \mathbf{e}_{p},
$$

where $j_{p} \in \tilde{N}_{p}$, and $\mathbf{e}_{p}$ is a "unit vector" along $p$. When adding such vectors, say $\mathbf{j}_{1}+\mathbf{j}_{2}$, one has to bear in mind that it should be performed componentwise, modulo $N_{p}$ for the component $p \in \pi(N)$.

The hidden symmetry group Aut $A$, i.e. the group of scalings, consists of elements

$$
\mathbf{r}=\sum_{p \in \pi(N)} r_{p} \mathbf{e}_{p}, \quad \operatorname{gcd}\left(r_{p}, N_{p}\right)=1
$$

and the action of $\mathbf{r}$ on $A$ is specified by

$$
\tau_{\mathbf{r}}=\left(\begin{array}{c}
\mathbf{j} \\
\mathbf{r j}
\end{array}\right), \quad \mathbf{j} \in A,
$$

where

$$
\mathbf{r j}=\sum_{p \in \pi(N)}\left(r_{p} j_{p} \bmod N_{p}\right) \mathbf{e}_{p} .
$$

Within this multidimensional realization of the cyclic $C_{N}$, some non-trivial scaling symmetries acquire a simple geometric meaning. We define

$$
\mathbf{i}_{p}=\mathbf{1}-2 \mathbf{e}_{p}, \quad p \in \pi(N),
$$

where $1 \in$ Aut $A$ is a unit scaling. It follows from Eq. (35) that

$$
\mathbf{i}_{p} \mathbf{e}_{p^{\prime}}=\left\{\begin{array}{cl}
\mathbf{e}_{p^{\prime}} & \text { for } p^{\prime} \neq p, \\
-\mathbf{e}_{p} & \text { for } p^{\prime}=p,
\end{array}\right.
$$

so that $\mathbf{i}_{p} \in$ Aut $A$ is a one-dimensional reflection of the multidimensional toroidal crystal. All these reflections generate a group

$$
E=\left\{\prod_{p \in \sigma} \mathbf{i}_{p} \mid \sigma \subset \pi(N)\right\} \subset \text { Aut } A
$$

with elements labelled by subsets $\sigma$ of the socle $\pi(N)$. E is the group of all geometric symmetries of the toroidal crystal, and its order is

$$
|E|= \begin{cases}2^{n-1} & \text { if } 2 \in \pi(N) \text { and } N_{2}=2, \\ 2^{n} & \text { otherwise }\end{cases}
$$

with $n=|\pi(N)|$ being the dimension of the crystal. 
Clearly, all dynamical scaling symmetries for $\mathbf{i} \in E$ acquire a transparent geometrical meaning. This element, written in the form

$$
\mathbf{i}=\prod_{p \in \sigma} \mathbf{i}_{p}
$$

for a subset $\sigma$ of the socle $\pi(N)$, corresponds to the inversion of all vectors $\mathbf{j}$ in the $n^{\prime}=|\sigma|$ - dimensional subspace of the toroidal crystal, with the vectors in the complement subspace corresponding to the set $\pi(N) \backslash \sigma$ left unchanged.

If we wish to compare geometric symmetries of the toroidal crystal with appropriate scalings of the linear ring, we need to fix the mapping $\beta: A \rightarrow C_{N}$, which should be consistent with the obvious symmetry of the recipe of Weyl, i.e. it should be an isomorphism of groups. The most general form of such a mapping is

$$
\beta(\mathbf{j})=\sum_{p \in \pi(N)} r_{p} j_{p} \bar{N}_{p} \bmod N,
$$

where $r_{p}, p \in \pi(N)$, define an automorphism $\mathbf{r} \in$ Aut $A$, and

$$
\bar{N}_{p}=N / N_{p}=\prod_{p^{\prime} \in \pi(N) \backslash\{p\}} N_{p^{\prime}}
$$

is the divisor complementary to $N_{p}$ in the lattice $K(N)$.

We observe that the decomposition (14) of the linear ring $\tilde{N}$ into orbits $\tilde{N}^{(\kappa)}$ of the hidden symmetry group Aut $C_{N}$ remains independent of the choice of the mapping $\beta$ given by Eq. (42), so that the corresponding scaling symmetry of the Hamiltonian (17) is preserved in the toroidal crystal too. Moreover, in this new realisation all scalings $\mathbf{i} \in E \subset$ Aut $\mathrm{A}$ acquire a geometric meaning of interactions between neighbours situated at the main diagonal in $n^{\prime}=|\sigma|$-dimensional subsocle consisting of all these $p \in \sigma \subset \pi(N)$ which undergo the inversion under $\mathbf{i} \in E$.

\section{An example for the clock-dial plate}

We demonstrate the dynamical symmetry for the case $N=12$, referred to as the clock dial plate. In this case we have

$$
\text { Aut } C_{12}=\{1,5,7,11\} \text {, }
$$

and Eq. (14) reads

$$
\widetilde{12}=\{1,5,7,11\} \cup\{2,10\} \cup\{3,9\} \cup\{4,8\} \cup\{6\} \cup\{12\},
$$

where the first element of each orbit $\tilde{N}^{(\kappa)}$ is the divisor $\kappa \in K(12)$. The lattice $K(12)$ is given in Fig. 1, and the socle is

$$
\pi(12)=\{2,3\} \subset K(12) .
$$




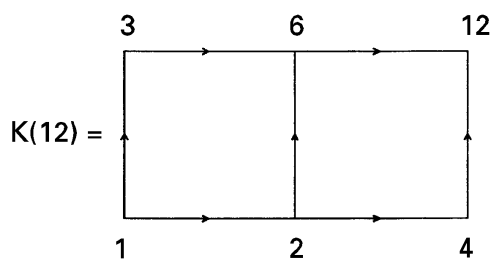

Fig. 1. The lattice $K(12)$.

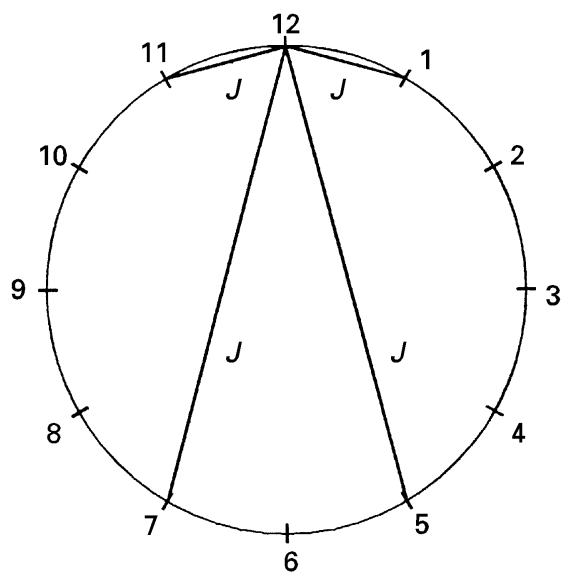

Fig. 2. Scaling-invariant exchange interactions in the clock dial plate (interactions of the node $j=12=0 \bmod 12$ with all the $\tilde{N}^{(\kappa)}$-neighbours for $N=12, \kappa=1$, are indicated).

The simplest scaling-invariant Hamiltonian, corresponding to $\kappa=12$ in Eq. (17), is defined by the exchange parameter $J$, the same for first $(\delta= \pm 1)$ and fifths $(\delta= \pm 5)$ neighbours, as shown in Fig. 2. The corresponding dispersion law for magnons (Eq. (29)) is presented in Fig. 3.

The canonical decomposition into primes,

$$
12=2^{2} \cdot 3
$$

yields a two-dimensional toroidal $4 \times 3$-periodic crystal, shown in Fig. 4 . We use here the isomorphism $\beta: C_{4} \times C_{3} \rightarrow C_{12}$, defined by the automorphism

$$
\mathbf{r}=3 \mathbf{e}_{2}+\mathbf{e}_{3}
$$

which corresponds to the canonical isomorphism of both obvious and hidden symmetry groups of the recipe of Weyl. In this two-dimensional realisation of the clock dial plate, the dynamical symmetry of the Heisenberg Hamiltonian under the scalings given by Eq. (44), corresponds to next-nearest-neighbour interactions, i.e. these along the diagonals $\mathbf{e}_{2} \pm \mathbf{e}_{3}$ of the toroidal crystal. 


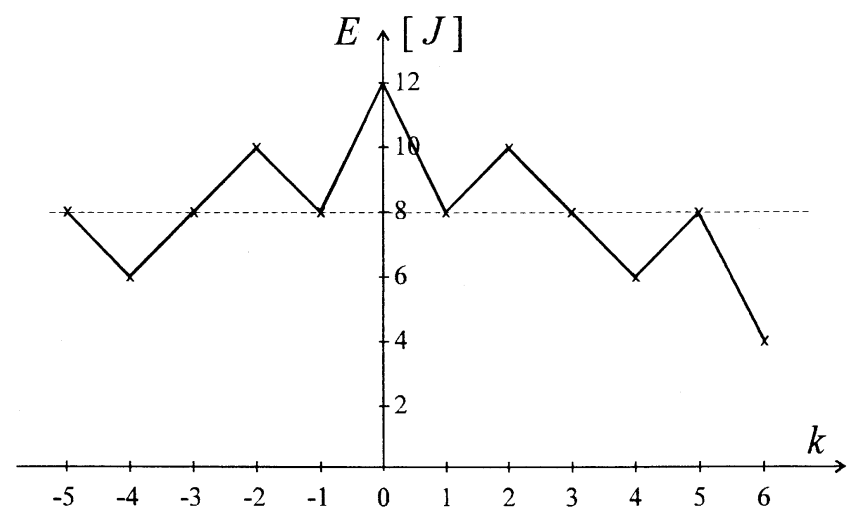

Fig. 3. Dispersion law for scaling-invariant magnons on the clock dial plate. $J$ denotes the exchange integral for $\kappa=12$. The dotted line corresponds to vanishing of the Möbius function. Values $k=0$ and 6 correspond respectively to the centre and the boundary of the Brillouin zone.

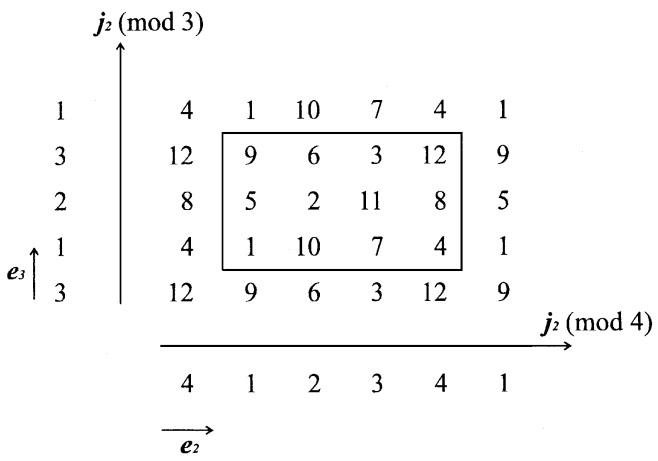

Fig. 4. The two-dimensional realisation of the clock-dial plate as a periodic toroidal crystal. The periodic unit is encircled by the $4 \times 3$-rectangle. The cartesian coordinates $\left(j_{2}, j_{3}\right)$ of a node of the crystal define the vector $\mathbf{j}=j_{2} \mathbf{e}_{2}+j_{3} \mathbf{e}_{3}$, with $j_{2} \in \tilde{4}$ and $j_{3} \in \tilde{3}$, and versors $\mathbf{e}_{2}$ and $\mathbf{e}_{3}$ shown in the figure. The point at the intersection $\left(j_{2}, j_{3}\right)$ is the corresponding value from the clock-dial plate, given by $j=\beta(\mathbf{j})=\left(9 j_{2}+4 j_{3}\right) \bmod 12 j \in \widetilde{12}$, and the associated group automorphism $\mathbf{r} \in$ Aut $A$ is given by $\mathbf{r}=3 \mathbf{e}_{2}+\mathbf{e}_{3}$ (cf. Eqs. 42 and 43). Scaling-invariant interactions defined by Fig. 2 correspond here to interactions of all nearest neighbours along both main diagonals of the coordinate system of Fig. 4, e.g. the node 1 interacts with $8,2,6$ and 12 , the node 12 - with $7,1,5$ and 11 , etc.

\section{Final remarks and conclusions}

We have proposed here a scaling-invariant version of the one-dimensional Heisenberg model of a magnet, with periodic boundary conditions. The scaling invariance of the kinematics of such a model has been already described as an essential feature of each model, which is independent upon the values of exchange integrals. Here we imposed this invariance as a dynamical symmetry, by requiring the exchange integrals to be constant on each orbit of the group Aut $C_{N}$ of scalings, acting on the linear chain $\tilde{N}$. We have shown that such a model implies a kind of "arithmetic magnons", with the 
dispersion law reproducing some values of Euler and Möbius functions of the number theory, related to the lattice $K(N)$ of all divisors of the number $N$ of nodes of the crystal. In the multidimensional realisation some scalings acquire a geometric meaning.

\section{Acknowledgements}

Two of us (Barbara Lulek and Tadeusz Lulek) would like to thank the Netherland Organisation for International Cooperation of Higher Education in the Netherlands for support, and the University of Twente in Enschede for hospitality and creation of fruitful scientific atmosphere. The support of Polish State Commitee for Scientific Research (KBN) by the grant No. 2 PO3B 11913 is also appreciated.

\section{References}

[1] B. Lulek, Acta Phys. Pol.22 (1991) 371-388.

[2] B. Lulek, Rarefied bands within the Heisenberg model of magnetism, in: W. Florek, T. Lulek, M. Mucha (Eds.), Symmetry and Structural Properties of Condensed Matter, World Scientific, Singapore, 1991.

[3] B. Lulek,J. Phys: Condens. Matter 4 (1992) 8737-8754.

[4] H. Weyl, Symmetry, Princeton University Press, New York, 1952.

[5] H. Hasse, Number Theory, Akademie Verlag, Berlin, 1979.

[6] W. Caspers, Spin Systems, World Scientific, Singapore, 1991.

[7] T. Lulek, M. Kuźma, R. Olchawa, Fractal symmetries of a linear chain, projections from multidimensional spaces, and the recipe of Weyl, in: T. Lulek, W. Florek, S. Wakcerz (Eds.), Symmetry and Structural Properties of Condensed Matter, World Scientific, Singapore, 1995.

[8] T. Lulek, R. Olchawa, J. Math. Phys. 37 (1996) 2472-2483.

[9] D. Loss, D.P. Di Vincenzo, G. Grinstein, D.D. Awschalom, J.F. Smyth, Physica B 189 (1993) 189-203.

[10] T. Lulek, J. Physique 45 (1984) 29-34.

[11] W. Florek, T. Lulek, J. Phys. A 20 (1987) 1921-1940. 